"This is the peer reviewed version of the following article: "Photochemical generation of radicals from alkyl electrophiles using a nucleophilic organic catalyst" which has been published in final form at https://doi.org/10.1038/s41557-018-0173-x. This article may be used for non-commercial purposes in accordance with Springer Nature Terms and Conditions for Self-Archiving."

\title{
Photochemical generation of radicals from alkyl electrophiles using a nucleophilic organic catalyst
}

\author{
Bertrand Schweitzer-Chaput ${ }^{1}$, Matthew A. Horwitz ${ }^{1}$,Eduardo de Pedro Beato ${ }^{1} \&$ Paolo Melchiorre ${ }^{1,2 *}$ \\ 1 - ICIQ, Institute of Chemical Research of Catalonia - the Barcelona Institute of Science and Technology, Av. Països Catalans 16 - 43007, Tarragona, Spain. \\ 2 - ICREA, Catalan Institution for Research and Advanced Studies, Passeig Lluís Companys 23 - 08010, Barcelona, Spain. \\ *email: pmelchiorre@iciq.es
}

Chemists extensively use free radical reactivity for applications in organic synthesis, materials science, and life science. Traditionally, generating radicals requires strategies that exploit the bond dissociation energy or the redox properties of the precursors. Here, we disclose a photochemical catalytic approach that harnesses different physical properties of the substrate to form carbon radicals. We use a nucleophilic dithiocarbamate anion catalyst, adorned with a well-tailored chromophoric unit, to activate alkyl electrophiles via an $S_{N} 2$ pathway. The resulting photon-absorbing intermediate affords radicals upon homolytic cleavage induced by visible light. This catalytic $S_{\mathrm{N}}$ 2-based strategy, which exploits a fundamental mechanistic process of ionic chemistry, grants access to open-shell intermediates from a variety of substrates that would be incompatible with or inert to classical radical-generating strategies. We also describe how the method's mild reaction conditions and high functional group tolerance could be advantageous for developing $\mathrm{C}$-C bond-forming reactions, for streamlining the preparation of a marketed drug, for the late-stage elaboration of biorelevant compounds, and for enantioselective radical catalysis.

Free radical chemistry offers powerful ways of making molecules that are often complementary to classical methods proceeding via ionic pathways ${ }^{1}$. This explains why radical processes have found application in material science, drug discovery, agrochemistry, and other disciplines ${ }^{2}$. Advances within the field have been spurred by the identification of effective radical-generating strategies. One traditional method relies on initiators ${ }^{3}$. Initiators are high-energy compounds (e.g. diazo compounds or peroxides), which, upon homolysis induced by heat or high-energy light, generate a reactive radical that can abstract a hydrogen or halogen atom from a substrate $\mathbf{S}$ (Fig. 1a, left panel). This approach ultimately relies on the bond dissociation energy (BDE) of $\mathbf{S}$ to form the target openshell intermediate $\mathbf{I}$. Another classical route to radicals exploits the tendency of a substrate $\mathbf{S}$ to engage in redox processes, which can be triggered by stoichiometric oxidants/reductants ${ }^{3}$ or by electrochemical means ${ }^{4}$ (Fig. 1a, right panel). Radical ions emerging from the single-electron transfer (SET) event can then fragment to yield the target radical I. These classical strategies are powerful. However, they require relatively harsh conditions, including hazardous and toxic reagents, high temperatures, and/or UV-light irradiation, overall limiting the selectivity and the functional group tolerance of the ensuing radical process.

A crucial step towards milder reaction conditions and, consequently, more selective reactions has been the use of substrates bearing thio functions and acting as both radical precursors and reactants (Fig. 1b, left). Methods introduced by Barton ${ }^{5-7}$ and further popularized by Zard with xanthate transfer chemistry ${ }^{8,9}$ greatly expanded the synthetic potential of radicals, but still relied on purposely designed stoichiometric reagents. Recently, photoredox catalysis has provided an attractive way to access radicals under very mild conditions and in a catalytic manner ${ }^{10-12}$. This approach exploits the ability of light-absorbing metal or organic catalysts to harness photonic energy to repeatedly remove an electron from or donate an electron to simple bench-stable substrates (Fig. 1b, right). This SET mechanism is generally used to produce the target radical $\mathbf{I}$, but some photoredox catalysts can also adopt a hydrogen abstraction manifold to generate $\mathbf{I}^{13}$. As with all other radical generation strategies, when applying photoredox catalysis a chemist must rely on the redox properties ${ }^{14}$ or the BDE to predict the suitability of a given substrate $\mathbf{S}$ to successfully form the target open-shell species $\mathbf{I}$.

Herein, we report a photochemical catalytic strategy that harnesses different physical properties of the substrates to generate carbon radicals (Fig. 1c). Specifically, we show that a nucleophilic dithiocarbonyl anion catalyst of type 1, adorned with a suitable chromophore, can undergo $\mathrm{S}_{\mathrm{N}} 2$ displacement of simple alkyl electrophiles, bearing different leaving groups. The resulting photonabsorbing intermediates II afford radicals upon excitation by weak visible light (blue light-emitting diodes, LEDs) and homolytic cleavage of the weak C-S bond. We used this $\mathrm{S}_{\mathrm{N}} 2$-based manifold to generate open-shell intermediates from a variety of substrates that would be inert to or incompatible with classical radical generating strategies. The method's mild reaction conditions and high functional group tolerance allowed the development of C-C bond-forming reactions, the streamlined preparation of a marketed drug, the late-stage derivatization of active pharmaceutical ingredients and biorelevant compounds, and enantioselective radical catalysis.

\section{Results and discussion}

Design Plan. Figure 2a details our proposed strategy to generate radicals by exploiting the electrophilic character of the precursors. We envisioned a catalytic cycle wherein a dithiocarbonyl anion $\mathbf{1}$ would serve as a nucleophilic catalyst that activates 
alkyl electrophiles 2 upon $\mathrm{S}_{\mathrm{N}} 2$ attack and displacement of the leaving group (LG). The resulting intermediate II possesses a weak $\mathrm{C}-\mathrm{S}$ bond (e.g. benzyl dimethyldithiocarbamate, where $\mathrm{R}=$ phenyl and $\mathrm{Z}=\mathrm{N}(\mathrm{Me})_{2}$, has a $\left.\mathrm{BDE}=31.3 \mathrm{kcal} / \mathrm{mol}\right)^{15}$, which could be cleaved by low-energy photons to generate the target carbon radical III and the dithiocarbonyl radical IV. The open-shell intermediate III would then be intercepted by an electron-deficient olefin $\mathbf{3}$ to furnish a new C-C bond. The emerging electrophilic radical $\mathbf{V}$ would in turn abstract a hydrogen atom from 1,4-cyclohexadiene (1,4-CHD), thus forming the final product $\mathbf{4}$ and the cyclohexadienyl radical VI. An exergonic SET reduction of the dithiocarbonyl radical IV (e.g. ethyl xanthogenate anion, where Z $=\mathrm{OEt}$, has reduction potential $\left.E_{\mathrm{red}}(\mathbf{I V} / \mathbf{1 a})=+0.04 \mathrm{~V} v s \mathrm{SCE}\right)^{16}$ from the cyclohexadienyl radical VI (the parent hydroxycyclohexadienyl radical has $E_{\text {red }}=-0.1 \mathrm{~V}$ vs SCE $)^{17}$ would eventually close the catalytic cycle by returning catalyst 1 . This mechanistic plan finds support in (i) the high nucleophilicity ${ }^{18}$ of dithiocarbonate and dithiocarbamate anions of type 1, (ii) a few studies establishing the tendency of thiocarbonyl compounds of type II to generate radicals upon photolytic cleavage ${ }^{19,20}$ and promote polymerizations (acting as photoiniferters) ${ }^{15}$, and (iii) the ability of 1,4-CHD to serve as a donor of both hydrogen atoms and electrons ${ }^{21}$.

Method optimization. To validate our proposal, we studied the Giese-type radical conjugate addition ${ }^{22}$ to dimethyl fumarate 3a (Fig. 2b) ${ }^{23-26}$. The experiments were conducted in acetonitrile $\left(\mathrm{CH}_{3} \mathrm{CN}\right)$ using commercially available $\gamma$-terpinene as a cheaper and more stable surrogate of 1,4-CHD. Our overarching goal was to define a strategy that permits the predictable and mild activation of substrates that would be inert to other practical catalytic approaches, including photoredox catalysis. We therefore selected benzyl chloride 2a, an industrial bulk chemical, as benzyl radical precursor. This choice was informed by the fact that $\mathbf{2 a}$ has a reduction potential $\left(E_{\mathrm{red}}\left(\mathbf{2} / \mathbf{2} \mathbf{a}^{-}\right)=-2.13 \mathrm{Vs} \mathrm{SCE}\right)^{27}$ sufficiently negative to remain out of reach of most commonly used photoredox catalysts $^{11,28}$. Therefore, SET reduction of $\mathbf{2 a}$ to generate the target benzyl radical would require strong stoichiometric reductants, greatly limiting its practicality and functional group compatibility. In contrast, benzyl chloride is an archetypical substrate for $\mathrm{S}_{\mathrm{N}} 2$ reactions ${ }^{29}$.

When conducting the model reaction using potassium ethyl xanthogenate 1a as nucleophilic catalyst (10 mol\%) and under 400 $\mathrm{nm}$ irradiation, the desired product $4 \mathbf{a}$ was obtained in $19 \%$ yield (entry 1, Fig. 2b). This initial result indicated the feasibility of catalytically generating the benzyl radical. The reaction was completely inhibited by changing the light source to a blue LED emitting at $465 \mathrm{~nm}$ (entry 2). We rationalized this reactivity behavior with the inability of the xanthate intermediate IIa, emerging from the $\mathrm{S}_{\mathrm{N}} 2$ process, to absorb blue light (Fig. 2c). The modular nature of the nucleophilic catalyst $\mathbf{1}$ allowed us to include a chromophoric unit, a structural modification aimed at enhancing the absorption properties of the key benzyl intermediate of type II. The thiocarbamate catalysts $\mathbf{1 b}$-c, bearing a carbazole and an indole scaffold, respectively, provided intermediates IIb-c upon $\mathrm{S}_{\mathrm{N}} 2$ reaction with substrate $\mathbf{2 a}$. These intermediates possess a dramatically increased molar extinction coefficient with respect to IIa. Pleasingly, those improved optical properties translated to a restored catalytic activity under blue light irradiation $(\lambda \max =465 \mathrm{~nm}$, entries 3 and 4). Raising the temperature to $60{ }^{\circ} \mathrm{C}$ brought the yield of product 3 to $90 \%$ when using the indole-containing thiocarbamate catalyst 1c (entry 5). During control experiments, no product formation was detected in the absence of catalyst $\mathbf{1}$ or light. The inhibition of the reactivity was also observed in the presence of the radical inhibitor 2,2,6,6-tetramethylpiperidine 1-oxyl (TEMPO, 1 equivalent), while using non-degassed conditions reduced the yield only slightly (entry 6). Mechanistically, we also evaluated the possibility that the formal dithiocarbamate group transfer product of type VII (depicted in Figure 2a) could be generated during the reaction. Although we never detected this adduct under catalytic conditions, an authentic sample of the VIItype intermediate afforded product $\mathbf{4 a}$ when irradiated with blue LEDs and in the presence of $\gamma$-terpinene. This observation, which is consonant with the tendency of dithiocarbonyl compounds towards group transfer manifolds, ${ }^{9,10}$ suggests that adduct VII can be a photoactive species in equilibrium with the progenitor radicals IV and $\mathbf{V}$.

As a testament to the method's robustness and relative tolerance to oxygen, the reaction could be efficiently performed in a simple test tube without degassing the solvent, and using water as co-solvent ( $83 \%$ yield, entry 7 ). The method's operational simplicity and robustness, in combination with the use of inexpensive reagents an air- and moisture-stable solid catalyst, make this reaction well-suited for multigram scale applications using commonly available glassware and light irradiation equipment (73\% yield, $8.6 \mathrm{~g}$, using simple blue LED strip, see Section D2 in the Supplementary Information for details). Notably, the solvent can be elected by considering the solubility of different substrates. No significant difference of reactivity was observed when performing the model reaction in a variety of reaction media (e.g. ethyl acetate, dichloroethane, toluene, and tetrahydrofuran all provided yields in the range of $85-90 \%$ ).

This strategy's underlying mechanism suggests that other benzylic substrates with leaving groups suitable for $\mathrm{S}_{\mathrm{N}} 2$ displacement could be used in the model reaction. Accordingly, different precursors 2b-e, bearing iodide, bromide, mesylate, or trifluoroacetate, afforded product 4a in high yields (Fig. 2d). The benzylamine-derived Katritzky salt $2 \mathbf{f}$ was also a suitable substrate. As such, this activation mode is not limited to halides, but amine and alcohol derivatives can also be used. In contrast, leaving groups with a poor tendency to undergo an $\mathrm{S}_{\mathrm{N}} 2$ process, including acetate and phosphate, remained completely unreacted. The choice of one leaving group or another can therefore be dictated by its ease of access or compatibility with other functional groups in a complex synthetic plan.

Scope of the radical generating strategy. We then evaluated the synthetic potential of this photochemical radical generation strategy in the context of different $\mathrm{C}$-C bond-forming transformations (Fig. 3). We first explored the scope of the Giese-type addition to fumarate 3a catalyzed by the nucleophilic catalyst 1c (Fig. 3a). A large variety of benzylic electrophiles bearing both electronrich and electron-poor aryl substituents were all competent substrates (2-j-j). In addition, a secondary benzylic substrate (2k) and prenyl chloride $\mathbf{2 l}$ (an allylic radical precursor) afforded the corresponding conjugate addition products in moderate yields. The strategy can be used to implement a radical $\alpha$-aminomethylation process, allowing the introduction of an oxazolidinone, a protected secondary amine, or a protected primary amine from readily available precursors $\mathbf{2} \mathbf{m}-\mathbf{o}$. We then evaluated the compatibility with 
unprotected polar functional groups, which is an essential criterion when assessing a method's potential applicability to complex molecule synthesis and drug discovery ${ }^{30}$. Our approach displayed a high level of tolerance towards nitrogen-containing heterocycles, including thiazole (2p), isoxazole (2q), pyrazole (2r), and triazole (2s) scaffolds. These common motifs are typically found in drug molecules. However, they generally represent a significant tolerability challenge for synthetic methods. In addition, our strategy's unique mechanism allowed the predictable and chemoselective activation of $S_{N} 2$-prone substrates bearing reactive functional groups, which would be incompatible with other radical generation methods or metal-based strategies. An aryl iodide (2t), a free carboxylic acid $(\mathbf{2 u})$, an unprotected alcohol $(\mathbf{2 v})$, and an aldehyde $(\mathbf{2 w})$ were all tolerated and activated exclusively at the desired benzylic position. Finally, we demonstrated that the Giese-type reaction of commercially available $N$-(chloromethyl)phthalimide 2o can be used with a variety of Michael acceptors (Fig. 3b). Olefins bearing assorted electron-withdrawing groups (e.g. esters, nitriles, sulfones, and imides) all reacted in good yields leading to adducts 5a-e. These functional groups create opportunities for product diversification. In general, the radical conjugate addition protocol requires weak light illumination and a slight excess (1.5 equivalents) of the radical precursor. Noteworthy, the choice of the leaving group in the radical precursor was dictated by commercial availability or ease of substrate synthesis.

We then applied our catalytic system to develop other synthetically useful radical C-C bond-forming processes. Based on our mechanistic proposal, we reasoned that a cyclohexadienyl radical of type VI, a crucial intermediate which acts as a reductant to turnover the catalyst (Fig. 2a), could be generated upon radical trapping by an electron-rich aromatic system. This mechanistic variation, which avoids the use of a stoichiometric $\mathrm{H}$ atom donor, was successfully implemented to enable the preparation of functionalized (hetero)aromatic products. The sequential radical addition/cyclization of aromatic acrylamides $\mathbf{6}^{31}$, promoted by catalyst 1c, provided a range of differently substituted oxindoles 7 (Fig. 3c). Similarly, the direct radical functionalization of electron-rich (hetero)aromatics, including an unprotected pyrrole (8i), was developed starting from easily available alkyl chlorides as radical precursors (Fig. 3d). Various reactive functional groups, including aldehydes, secondary amides, and free alcohols, were tolerated, leading to synthetically valuable substituted (hetero)aromatic compounds $\mathbf{8}^{32}$.

To showcase our system's synthetic utility, this strategy was applied to the synthesis of the pyrrole derivative $\mathbf{8 h}$ (Fig. 4a). Adduct $\mathbf{8 h}$ is an intermediate en route to Tolmetin, a marketed nonsteroidal anti-inflammatory drug ${ }^{33,34}$. Using 5 mol\% of catalyst 1c, we could perform a multigram-scale preparation of $\mathbf{8 h}$ in ethyl acetate, an industrially preferred solvent. The reaction was also amenable to the use of a continuous-flow photoreactor ${ }^{35}$. Further experiments detailed in Fig. $4 \mathrm{~b}$ demonstrated that this method is compatible with the functionalization of biorelevant compounds bearing unprotected polar functional groups. It allowed the latestage introduction of a pyrrole (adduct 10) and an oxindole moiety (11) into mesylate derivative $\mathbf{9}$, synthesized in a single step from cortisone. The nucleobase analogues $\mathbf{1 3}$ and $\mathbf{1 6}$ could be readily obtained from commercially available uracyl chloride $\mathbf{1 2}$ and adenine 14, respectively.

Finally, we applied our strategy to perform an organocatalytic stereoselective intermolecular $\alpha$-alkylation of aldehydes using alkyl chlorides. This transformation, which was originally reported in $2008^{36}$ using bromomalonate 18a and the chiral secondary amine catalyst 19a to afford the enantioenriched product 20 (Fig. 5a), laid the foundations for the development of the field of photoredox catalysis ${ }^{11,37}$. Since then, this process has served as a benchmark to measure the efficiency of new photoredox catalysts $^{38,39}$. All procedures reported so far used alkyl bromides as radical precursors. As depicted in Fig. 5b, the dithiocarbamate anion catalyst 1c can effectively activate a variety of easily available alkyl chlorides toward the direct enantioselective alkylation of aldehydes 17. The chemistry was effectively performed at ambient temperature using $\mathbf{1 c}$ in combination with organocatalysts 19a or $\mathbf{1 9 b}$ to afford the corresponding $\alpha$-alkylation products $\mathbf{2 0 - 2 2}$.

\section{Conclusions}

In summary, we have reported a photochemical strategy that exploits an $\mathrm{S}_{\mathrm{N}} 2$ process (a fundamental path in ionic chemistry ${ }^{29}$ ) to generate open-shell intermediates from a variety of electrophilic substrates that would be incompatible with or inert to traditional radical-generating strategies. The method requires a readily available organic catalyst and occurs under visible-light irradiation. Substrate scope investigations indicate that unprotected polar functional groups and $N$-heterocycles are all well-tolerated. Preliminary results highlight that this radical-generation strategy can be used in more complex settings, including light-driven enantioselective catalysis ${ }^{40}$. These findings, along with the experimental simplicity and the low cost of the catalyst, suggest that this method will find further synthetic applications and is potentially amenable to medicinal chemistry and process development.

Data availability The data supporting the findings of this study are available within the paper and its Supplementary Information. Crystallographic data for an analogue of the major diastereoisomer of compound $\mathbf{1 1}$ have been deposited with the Cambridge Crystallographic Data Centre, accession number CCDC 1839232.

\section{References}

1. Radicals in Organic Synthesis, Renaud, P. \& Sibi, M. P. Eds., Wiley-VCH: Weinheim, Germany, 2001.

2. Yan, M., Lo, J. C., Edwards, J. T. \& Baran, P. S. Radicals: reactive intermediates with translational potential. J. Am. Chem. Soc. 138, 12692-12714 (2016).

3. Lalevée, J. \& Fouassier, J. P. "Overview of radical initiation" in Encyclopedia of Radicals in Chemistry, Biology and Materials John Wiley \& Sons, 2012, vol. 1, pp 37-56.

4. Yan, M., Kawamata, Y. \& Baran, P. S. Synthetic organic electrochemical methods since 2000: on the verge of a renaissance. Chem. Rev. 117, 13230-13319 (2017). 
5. Barton, D. H. R. \& McCombie, S. W. A new method for the deoxygenation of secondary alcohols. J. Chem. Soc. Perkin Trans. 1, 1574-1585 (1975).

6. Barton, D. H. R., Crich, D. \& Motherwell, W. B. New and improved methods for the radical decarboxylation of acids. J. Chem. Soc. Chem. Commun. 939-941 (1983).

7. Barton, D. H. R. \& Zard, S. Z. Invention of new reactions useful in the chemistry of natural products. Pure Appl. Chem. 58, 675-684 (1986).

8. Delduc, P., Tailhan, C. \& Zard, S. Z. A convenient source of alkyl and acyl radicals. J. Chem. Soc., Chem. Commun. 308-310 (1988).

9. Zard, S. Z. On the trail of xanthates: some new chemistry from an old functional group. Angew. Chem. Int. Ed. 36, 672-685 (1997).

10. Studer, A. \& Curran, D. P. Catalysis of radical reactions: a radical chemistry perspective. Angew. Chem. Int. Ed. 55, 58-102 (2016).

11. Shaw, M. H., Twilton, J. \& MacMillan, D. W. C. Photoredox catalysis in organic chemistry. J. Org. Chem. 81, 6898-6926 (2016).

12. Matsui, J. K., Lang, S. B., Heitz, D. R. \& Molander, G. A. Photoredox-mediated routes to radicals: the value of catalytic radical generation in synthetic methods development, ACS Catal. 7, 2563-2575 (2017).

13. Tzirakis, M. D., Lykakis, I. N. \& Orfanopoulos, M. Decatungstate as an efficient photocatalyst in organic chemistry. Chem. Soc. Rev. 38, 2609-2621 (2009).

14. Roth, H. G., Romero, N. A. \& Nicewicz, D. A. Experimental and calculated electrochemical potentials of common organic molecules for applications to single-electron redox chemistry. Synlett 27, 714-723 (2016).

15. Lalevée, J., Blanchard, N., El-Roz, M., Allonas, X. \& Fouassier, J. P. New photoiniferters: respective role of the initiating and persistent radicals. Macromolecules 41, 2347-2352 (2008).

16. Dag, O., Yaman, S. O., Onal, A. M. \& Isci, H. Spectroelectrochemistry of potassium ethylxanthate, bis(ethylxanthato)nickel(II) and tetraethylammonium tris(ethylxanthato)nickelate(II). J. Chem. Soc., Dalton Trans. 2819-2824 (2001).

17. Bahtia, K. \& Schuler, R. H. Oxidation of hydroxycyclohexadienyl radical by metal ions. J. Phys. Chem. 78, 2335-2338 (1974).

18. Duan, X.-H., Maji, B. \& Mayr, H. Characterization of the nucleophilic reactivities of thiocarboxylate, dithiocarbonate and dithiocarbamate anions. Org. Biomol. Chem. 9, 8046-8050 (2011).

19. Barton, D. H. R., George, M. V. \& Tomoeda, M. 369. Photochemical transformations. Part XIII. A new method for the production of acyl radicals. J. Chem. Soc., 1967-1974 (1962).

20. Grainger, R. S. \& Welsh, E. J. Formal synthesis of (-)-Aphanorphine using sequential photomediated radical reactions of dithiocarbamates. Angew. Chem. Int. Ed. 46, 5377-5380 (2007).

21. Davies, J., Svejstrup, T. D., Fernandez Reina, D., Sheikh, N. S. \& Leonori, D. Visible-light-mediated synthesis of amidyl radicals: transition-metal-free hydroamination and N-arylation reactions. J. Am. Chem. Soc. 138, 8092-8095 (2016).

22. Giese, B. Formation of C-C bonds by addition of free radicals to alkenes. Angew. Chem. Int. Ed. Int. 22, 753-764 (1983).

23. Proinsias, K. ó, Jackowska, A., Radzewicz, K., Giedyk, M. \& Gryko, D. Vitamin B ${ }_{12}$ catalyzed atom transfer radical addition. Org. Lett. 20, 296-299 (2018).

24. Gualandi, A., Mazzarella, D., Ortega-Martínez, A., Mengozzi, L., Calcinelli, F., Matteucci, E., Monti, F., Armaroli, N., Sambri, L. \& Cozzi, P. G. Photocatalytic radical alkylation of electrophilic olefins by benzylic and alkylic zinc-sulfinates. ACS Catal. 7, 5357-5362 (2017).

25. Ueda, M., Kondoh, E., Ito, Y., Shono, H., Kakiuchi, M., Ichii, Y., Kimura, T., Miyoshi, T., Naito, T., Miyata, O. Benzyl radical addition reaction through the homolytic cleavage of a benzylic C-H bond. Org. Biomol. Chem. 9, 2062-2064 (2011).

26. Capaldo, L., Buzzetti, L., Merli, D., Fagnoni, M. \& Ravelli, D. Smooth photocatalyzed benzylation of electrophilic olefins via decarboxylation of arylacetic acids, J. Org. Chem. 81, 7102-7109 (2016).

27. Isse, A. A., Falciola, L., Mussini, P. R. \& Gennaro, A. Relevance of electron transfer mechanism in electrocatalysis: the reduction of organic halides at silver electrodes. Chem. Commun. 344-346 (2006).

28. Brasholz, M. "Super-reducing" photocatalysis: consecutive energy and electron transfers with polycyclic aromatic hydrocarbons. Angew. Chem. Int. Ed. 56, 10280-10281 (2017).

29. Clayden, J., Greeves, N., Warren, S. \& Wothers, P. Eds. Organic Chemistry, chapter 17, pp 407-445 (Oxford University Press, 2001).

30. Blakemore, D. C., Castro, L., Churcher, I., Rees, D. C., Thomas, A. W., Wilson, D. M. \& Wood, A. Organic synthesis provides opportunities to transform drug discovery. Nat. Chem. 10, 383-394 (2018).

31. Chen, J.-R., Yu, X.-Y. \& Xiao, W.-J. Tandem radical cyclization of N-arylacrylamides: an emerging platform for the construction of 3,3-disubstituted oxindoles. Synthesis 47, 604-629 (2015).

32. Fujiwara, Y. \& Baran, P. S. "Radical-based late stage C-H functionalization of heteroaromatics in drug discovery" in New Horizons of Process Chemistry, Springer Nature Singapore Pte Ltd., 2017, pp 103-120.

33. Carson, J. R. Uncatalyzed aroylation of 1-alkylpyrrole-2-acetic acid derivatives, U.S. Patent US3998844A (1976).

34. Liu, Z.-Q. \& Li, Z. Radical-promoted site-specific cross dehydrogenative coupling of heterocycles with nitriles. Chem. Commun. 52, 14278-14281 (2016).

35. Cambié, D., Bottecchia, C., Straathof, N. J. W., Hessel, V. \& Noël, T. Applications of continuous-flow photochemistry in organic synthesis, material science, and water treatment. Chem. Rev. 116, 10276-10341 (2016).

36. Nicewicz, D. \& MacMillan, D. W. C. Merging photoredox catalysis with organocatalysis: the direct asymmetric alkylation of aldehydes. Science 322, 77-80 (2008).

37. Silvi, M. \& Melchiorre P. Enhancing the potential of enantioselective organocatalysis with light. Nature 554, 41-49 (2018).

38. Neumann, M., Füldner, S., König, B. \& Zeitler, K. Metal-free, cooperative asymmetric organophotoredox catalysis with visible light. Angew. Chem. Int. Ed. 50, 951-954 (2011).

39. Gualandi, A., Marchini, M., Mengozzi, L., Natali, M., Lucarini, M., Ceroni, P. \& Cozzi, P. G. Organocatalytic enantioselective alkylation of aldehydes with [Fe(bpy $\left.)_{3}\right] \mathrm{Br}_{2}$ catalyst and visible light. ACS Catal. 5, 5927-5931 (2015).

40. Brimioulle, R., Lenhart, D., Maturi, M. M. \& Bach, T. Enantioselective catalysis of photochemical reactions. Angew. Chem. Int. Ed. 54, 3872-3890 (2015).

Acknowledgements We thank ICIQ, MINECO (CTQ2016-75520-P and Severo Ochoa Accreditation SEV-2013-0319), and the European Research Council (ERC 681840 - CATA-LUX) for financial support.

Author contributions B.S.-C. was involved in the discovery and initial development of the radical generation strategy. B.S.-C., M.A.H., and E.P.B. performed the experiments. All the authors analyzed the data and designed the experiments. P.M directed the project and wrote the manuscript with contributions from all the authors. 
Additional information Supplementary information and chemical compound information are available in the online version of the paper. Reprints and permissions information is available online at www.nature.com/reprints. Correspondence and requests for materials should be addressed to P.M. (pmelchiorre@iciq.es).

Competing financial interests The authors declare no competing financial interests.

\section{Figure Legends}

Figure 1 | Strategies for generating radicals. a, Established atom abstraction methods (left panel) generally rely on initiators, which are high-energy compounds, and require precursors with suitable bond-dissociation energies (BDE). Electron transfer strategies use stoichiometric reductants/oxidants or electrochemical apparatus and require precursors with suitable redox properties. b, More efficient approaches to generate radicals under milder conditions using stoichiometric precursors and photoredox catalysts. c, $S_{\mathrm{N}} 2$-based photochemical catalytic protocol for generating radicals from precursors that are redox-inactive or not prone to undergoing atom abstraction activation. SET, single-electron transfer; LG, leaving group.

Figure 2 | Design plan and reaction development. a, Proposed catalytic cycle. b, The model reaction and the nucleophilic catalysts evaluated. c, Absorption of intermediates II, recorded in $\mathrm{CH}_{3} \mathrm{CN}$ at $[0.05 \mathrm{M}]$, isolated upon $\mathrm{S}_{\mathrm{N}} 2$ reaction of catalysts 1 and benzyl chloride 2a. d, Evaluating the leaving groups (LG) suitable for the $S_{N} 2$-based benzyl radical generation strategy.

Figure 3 | Reaction Scope. Reactions performed at $0.5 \mathrm{mmol}$ scale, unless otherwise stated; yields refer to isolated material after purification. a, Radical precursors suitable for the Giese addition to dimethyl fumarate 3a. Functional groups that are prone to atom abstraction (products $\mathbf{2 t}, \mathbf{2 v}$, and $\mathbf{2 w}$ ) and to SET oxidation (adduct $\mathbf{2 u}$ ) or are incompatible with metal catalysts (2t), bases (2u), and organometallic reagents $(\mathbf{2 u}$ and $\mathbf{2 w})$ are tolerated well. $\mathbf{b}$, Scope of the electron-poor olefins for the Giese addition. $\mathbf{c}$, Tandem radical addition/cyclization of $\mathrm{N}$-arylacrylamides affording 3,3-disubstituted oxindoles. d, Radical functionalization of electron-rich (hetero)arenes. Ms, mesylate; DCE, 1,2-dichloroethane; NPhth, phthalimide. *Run over 48 hours using 20 equiv. of $\mathrm{H}_{2} \mathrm{O}$. ${ }^{\dagger} 2$ equivalents of $\mathbf{2}, \gamma$-terpinene, and 2,6-lutidine. $\$ 3$ equivalents of $\mathbf{2}, \gamma$-terpinene, and 2,6-lutidine. $\# 2$ equivalents of $\gamma$-terpinene and 2,6-lutidine. **NMR yield. ${ }^{\dagger}$ Using bromine as the leaving group.

Figure 4 | Method's synthetic applications. Streamlined preparation of a marketed drug (a) and late-stage elaboration of biorelevant compounds (b). The stereochemistry of the major diastereoisomer of compound $\mathbf{1 1}$ was inferred by X-ray analysis (CCDC 1839232). Prices of reagents determined according to Sigma-Aldrich catalogue at the highest quantity available; *NMR yield.

Figure 5 | Application in enantioselective radical catalysis. a, The catalytic asymmetric $\alpha$-alkylation of aldehydes using a visiblelight-activated ruthenium catalyst $\left(\mathrm{Ru}(\mathrm{bpy})_{3}{ }^{2+}\right)$. The chemistry uses alkyl bromide derivatives. b, Demonstration that the dithiocarbamate anion catalytic strategy can extend the asymmetric alkylation of aldehydes to include alkyl chlorides. Bpy: 2,2'bipyridine; $\mathrm{CFL}=$ compact fluorescence lamp; SET = single-electron transfer; TMS: trimethylsilyl.

\section{Table of Contents Summary}

A photochemical strategy is reported that exploits an $S_{N} 2$ process -a fundamental ionic path- to generate radicals from alkyl electrophiles: the method occurs readily under visible-light irradiation, exhibits broad functional group tolerance, and grants access to open-shell intermediates from substrates that would be incompatible with or inert to traditional radical-generating strategies. 
$\mathrm{R}-\mathrm{H}$

substrate $\mathbf{S}$
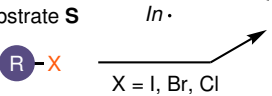

atom abstraction mechanism

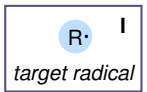

SET reduction

substrate $\mathbf{S}$

\section{electron-transfer mechanism}

SET mechanism

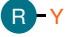

R

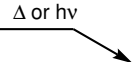

R. I

target radical

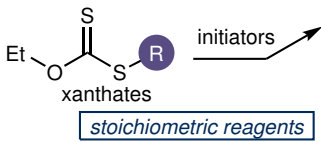

c this work $\mathrm{R}-\mathrm{LG}$
substrate
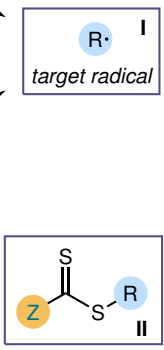

photon-absorbing intermediate

turnover event
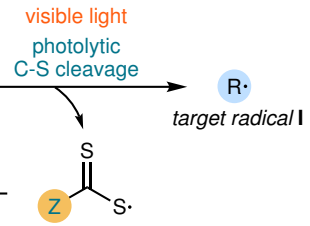
a 2008 (Ref. 36)

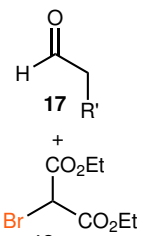

18a

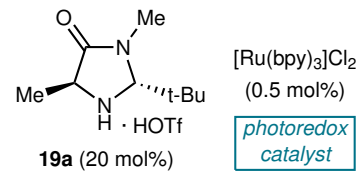

15 W CFL

2,6-lutidine, DMF

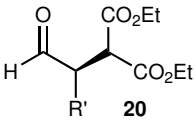

organic catalyst 19 (20 mol\%) dithiocarbamate catalyst 1c (20 mol\%)

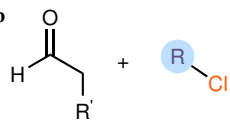

2,6-lutidine (1.2 equiv)

blue LEDs, MeCN, 24 h, $25^{\circ} \mathrm{C}$

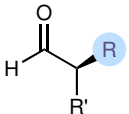

17

catalysts

19b

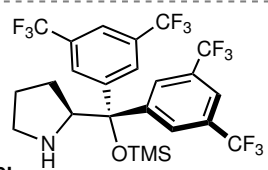

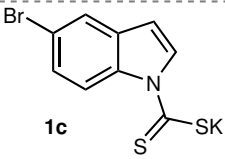

dithiocarbamate catalyst

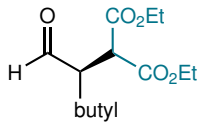

20: $77 \%$ y, $74 \%$ ee (using catalyst 19b)

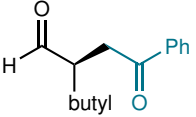

21: $57 \%$ y, $79 \%$ ee (using catalyst 19b)

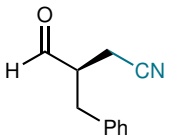

22: $60 \%$ y, $90 \%$ ee (using catalyst 19a) 


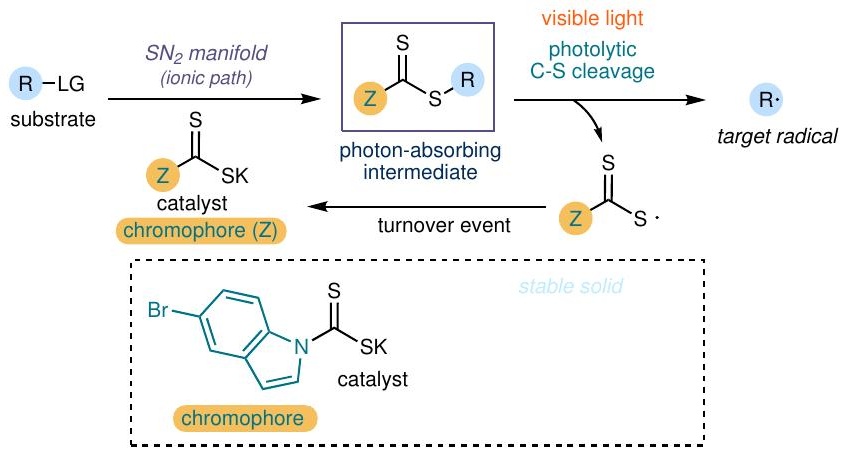

